\title{
Bilateral lung transplantation (BLTx) in a 9-year-old girl with bronchopulmonary dysplasia with pulmonary hypertension
}

\author{
Chiharu Ota ${ }^{1}$, Ryoko Saito ${ }^{1}$, Junya Tominaga ${ }^{2}$, Shinya Iwasawa ${ }^{2}$, Hirama Takashi², \\ Yasushi Matsuda ${ }^{2}$, Katsunori Ono ${ }^{2}$, Takehiko Onoki ${ }^{2}$, Masato Kimura ${ }^{3}$, Yoshinori \\ Kawabata $^{4}$, and Yoshinori Okada ${ }^{2}$ \\ ${ }^{1}$ Tohoku University Graduate School of Medicine \\ ${ }^{2}$ Tohoku University Hospital \\ ${ }^{3}$ Miyagi Children's Hospital \\ ${ }^{4}$ Saitama Cardiovascular and Respiratory Center
}

March 18, 2021

\begin{abstract}
Background Bronchopulmonary dysplasia (BPD) is a chronic respiratory disease that occurs in premature infants and the prognosis is variable depending on the comorbidities including fibrosis, emphysema, or pulmonary hypertension (PH). We present a case of a 9-year-old girl who developed PH associated with severe BPD (BPD-PH) and underwent bilateral lung transplantation (BLTx). Case description A 9-year-old girl admitted to our department to undergo BLTx. She was born at 23 weeks and 4 days gestation with a weight of 507 grams. She received ventilation for the first 2 months and required further respiratory care due to repetitive, severe respiratory infections. She was diagnosed with BPD-PH at 6 months of age and oral administration of pulmonary vasodilators were initiated. She was registered as a lung transplant candidate at 4 years of age after the life-threatening exacerbation. Chest computed tomography (CT) revealed severe lung conditions with groundglass opacities and emphysematous low-density areas in the upper and lower lobes. BLTx from a brain-dead male donor was performed. The pathological findings of her resected lung revealed saccular, hypoplastic lung with alveolar repair/regeneration and medial hypertrophy and muscularization of peripheral arteries. The postoperative course was mostly uneventful. She was free from oxygen administration and showed no signs of PH after 6 months of the surgery. Conclusion This is the first case report of BLTx in a pediatric, irreversible BPD-PH patient with detailed pathohistological findings and clinical examination. Lung transplantation is one of the treatment options for severe BPD-PH.
\end{abstract}

Title: Bilateral lung transplantation (BLTx) in a 9-year-old girl with bronchopulmonary dysplasia with pulmonary hypertension

Chiharu Ota ${ }^{1}$, Ryoko Saito ${ }^{2}$, Junya Tominaga ${ }^{3}$, Shinya Iwasawa ${ }^{1}$, Takashi Hirama ${ }^{4}$, Yasushi Matsuda ${ }^{4}$, Katsunori $\mathrm{Ono}^{3}$, Takehiko Onoki ${ }^{1}$, Masato Kimura ${ }^{5}$, Yoshinori Kawabata ${ }^{6}$, Yoshinori Okada ${ }^{4}$

${ }^{1}$ Department of Pediatrics, Tohoku University Hospital, Sendai, Japan,

${ }^{2}$ Department of Pathology, Tohoku University Graduate School of Medicine, Sendai, Japan

${ }^{3}$ Department of Diagnostic Radiology, Tohoku University Hospital, Sendai, Japan

${ }^{4}$ Department of Thoracic Surgery, Institute of Development, Aging and Cancer, Tohoku University Hospital, Sendai, Japan

${ }^{5}$ Department of Pediatric Cardiology, Miyagi Children's Hospital, Sendai, Japan 
${ }^{6}$ Division of Diagnostic Pathology, Saitama Cardiovascular and Respiratory Center, Saitama, Japan

Corresponding author: Chiharu Ota

E-mail: chiharu.ota.e8@tohoku.ac.jp

\section{Introduction}

Bronchopulmonary dysplasia (BPD) is a chronic respiratory disease occurring in premature infants due to inflammation, oxygen supplementation, and/or prolonged mechanical ventilation. Eighty percent of the patients show "reversible" BPD with improving respiratory status within 2 years after birth while $20 \%$ show "irreversible" BPD with complicated comorbidities including pulmonary hypertension $(\mathrm{PH})^{1}$. We present a case of a 9-year-old girl who developed PH associated with severe BPD (BPD-PH) and underwent bilateral lung transplantation (BLTx).

\section{Case description}

A 9-year-old girl was admitted to our department for BLTx. She was born at 23 weeks and 4 days of gestation with a weight of 507 grams. She received mechanical ventilation for the first 2 months and required further respiratory care with tracheostomy. She was diagnosed with BPD-PH at 6 months of age using transthoracic echocardiography. The oral administration of sildenafil, a phosphodiesterase- 5 inhibitor, and beraprost, a prostacyclin analogue, was initiated. At 2 years and 6 months of age, right heart catheterization (RHC) showed an elevated mean pulmonary arterial pressure (mPAP) of $49 \mathrm{mmHg}$, a pulmonary capillary wedge pressure (PCWP) of $12 \mathrm{mmHg}$, and a high pulmonary vascular resistance (Rp) of 13 wood units. Pulmonary angiography (PAG) revealed hypoplastic pulmonary arteries and dilated main pulmonary artery (Figure 1A). At 4 years of age, she experienced transient cardiopulmonary arrest (CPA) caused by the exacerbation of $\mathrm{PH}$ with severe respiratory infection. RHC revealed rapid aggravation of $\mathrm{PH}, \mathrm{mPAP}$ of $70 \mathrm{mmHg}, \mathrm{PCWP}$ of $8 \mathrm{mmHg}$, and Rp of 22 wood units with hypoplastic pulmonary arteries by PAG (Figure 1B). Subsequently, she was listed as a candidate for lung transplantation at that time. An endothelin receptor antagonist was added to the treatment regimen and her respiratory condition was again gradually stabilized for the following years with familial support to prevent respiratory infections. Nevertheless, a follow-up RHC at 8 years old demonstrated $\mathrm{PH}$ with mPAP of $32 \mathrm{mmHg}$, PCWP of $8 \mathrm{mmHg}$, and Rp of 8 wood units with hypoplastic pulmonary arteries by PAG (Figure 1C). Chest computed tomography (CT) revealed severe lung conditions with ground-glass opacities and emphysematous low-density areas (Figure 1D). In addition, pulmonary perfusion scintigraphy revealed low blood flow in the bilateral lower lobes (Figure 1E). When she was 9 years and 7 months, the lungs from a brain-dead male donor under 6 years of age were donated.

On admission, she weighed $21.6 \mathrm{~kg}$ (-1.5 standard deviation; SD), was $113.5 \mathrm{~cm}$ (-3.2 SD) tall, and her oxygen saturation was $95 \%$ with $3 \mathrm{~L} / \mathrm{min}$ of oxygen supplementation through a tracheostomy. BLTx was performed and the immediate postoperative course was mostly uneventful. She was free from oxygen supplementation when she was discharged from our department. Figure $1 \mathrm{~F}$ shows lung growth by CT-based lung volumetric analysis from 1 years old to 10 years old, before and after BLTx. Figure 1G depicts the lung volume divided by height, showing that the lung volume/height increased after the CPA episode and reached a plateau thereafter.

We performed a detailed pathological examination of the patient's lungs extracted during the surgery. Macroscopically, emphysema-like cystic changes of up to $2 \mathrm{~cm}$ and patchy mild solid appearance were observed in several areas (Figure 1H). The emphysema-like areas demonstrated dilated alveolar spaces with thin distracted alveolar septa without apparent epithelial injury or repair such as fibrosis (Figure 1I). In contrast, the solid areas showed thickened alveolar walls with fibrosis, chronic inflammatory cell infiltration, foreign body reaction and hypertrophic smooth muscle, suggesting chronic epithelial injury and repair (Figure 1J, $\mathrm{K}$ ). In addition, the muscularization of the peripheral arteries and mild medial hypertrophy, compatible with Heath-Edwards classification Grade $1 \mathrm{PH}$, were observed especially in the area showing fibrosis (Figure 1L). In addition, capillary proliferation was observed in the alveolar septa (Figure 1M). No apparent occlusion of 
veins or venules was detected.

\section{Discussion}

In our case, along with the premature lungs at the canalicular stage of lung

development, prolonged ventilation and repetitive respiratory infections may cause BPD-PH. Pulmonary hypoplasia due to premature development of the lung is one of the causes of pediatric $\mathrm{PH}^{2}$. Pulmonary angiography revealed hypoplastic peripheral pulmonary arteries and a dilated main pulmonary artery (Figure 1A-C). Additionally, pulmonary perfusion scintigraphy showed reduced pulmonary blood flow in the bilateral lower lungs (Figure 1E). Capillary proliferation shown in pathological findings (Figure 1M) can be explained as a compensatory response for hypoplastic vascular bed ${ }^{3}$. It is also possible that treatment with pulmonary vasodilators promoted vascular growth ${ }^{4}$. On the other hand, chest CT showed a combination of fibrotic areas and emphysematous low-density areas with several distinct bullae at the upper and lower lobes. Along with the above mechanisms, lung parenchymal destruction and interstitial inflammation or fibrosis cause PH due to reduction of the vascular bed and subsequent hypoxic pulmonary vasoconstriction. Notably, CT-based lung volume estimation showed that the lung volume had been constantly growing (Figure $1 \mathrm{~F}$ ), and that after the CPA episode, the lung volume/height ratio had expanded and subsequently plateaued until BLTx (Figure $1 \mathrm{G}$ ). We speculate that the high positive airway pressure ventilation during CPA resuscitation caused the exacerbation of emphysematous changes in the patient's vulnerable lungs.

We histologically diagnosed the patient's lung with pulmonary hypoplasia and BPD-PH. Microscopically, the lung demonstrated hypoplastic alveoli resembling a saccular-stage lung, in which the radial alveolar count was only 3-4 (normal range: >5-6 after birth) and only a few alveolar entrance rings were formed (Figure $1 \mathrm{~N})$. There was no mismatch in the development of the airway and blood vessels.

These pathological findings revealed that pulmonary hypoplasia due to ceased lung development at the saccular stage led to BPD-PH. In addition, there were various irreversible changes in the lungs, including emphysema-like cystic changes, which may be due to the fragility associated with extension because there was no evidence of epithelial injury like adult chronic obstructive pulmonary disease. As described above, CT-based lung volume estimation also supports these findings. In contrast, the fibrotic changes with chronic epithelial injury and alveolar repair may be due to repetitive inflammation such as infection, hypo/hyperoxia, or ventilation-associated lung injury.

In conclusion, we showed a case of BLTx in a pediatric, irreversible BPH-PH patient with detailed pathohistological findings and clinical examination. LTx will be one of the options for pediatric severe BPD-PH patients as reported recently for the adult BPD-PH patients ${ }^{5}$.

1. Cristea AI, Carroll AE, Davis SD, Swigonski NL, Ackerman VL. Outcomes of Children With Severe Bronchopulmonary Dysplasia Who Were Ventilator Dependent at Home. Pediatrics 2013;132(3):e727-e734.

2. Rosenzweig EB, Abman SH, Adatia I, Beghetti M, Bonnet D, Haworth S, Ivy DD, Berger RMF. Paediatric pulmonary arterial hypertension: updates on definition, classification, diagnostics and management. Eur Respir J 2019;53(1).

3. Saito R, Kasajima A, Kawabata Y, Miki Y, Tominaga J, Tatebe S, Nakajima H, Hata S, Inoue C, Taniuchi $\mathrm{S}$, et al. Vasohibin-1 and miR-720 expression in diffuse pulmonary capillary hemangiomatosis-like changes associated with pulmonary hypoplasia. Pathol Int 2020;70(7):470-472.

4. Mous DS, Kool HM, Wijnen R, Tibboel D, Rottier RJ. Pulmonary vascular development in congenital diaphragmatic hernia. Eur Respir Rev 2018 [accessed 2020 Oct 14];27(147). https://err.ersjournals.com/content/27/147/170104

5. Liu N, Cummings OW, Lagstein A, Hage CA, Chan KM, Zhang C. Lung Transplantation for Bronchopulmonary Dysplasia in Adults: A Clinical and Pathologic Study of 3 Cases. Am J Surg Pathol 2020;44(4):509515 . 

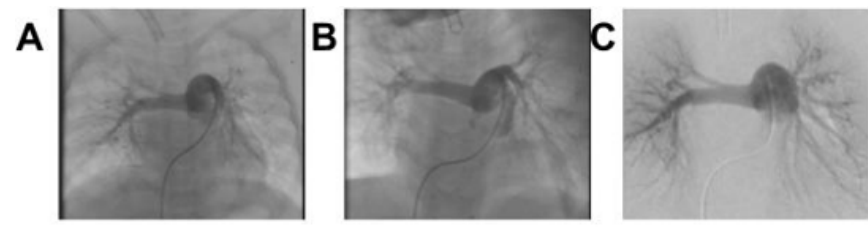

D

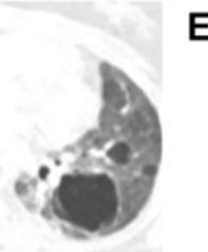

E
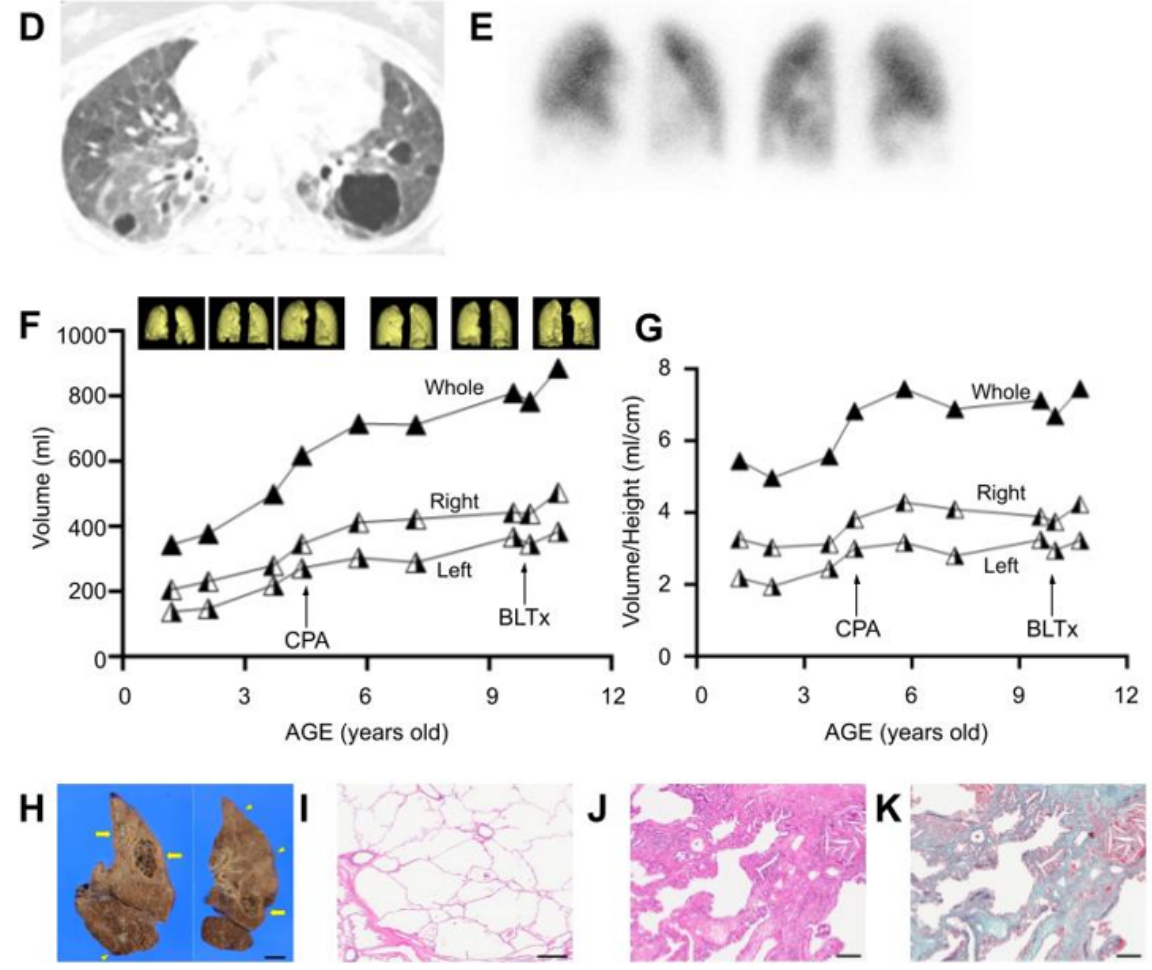

L
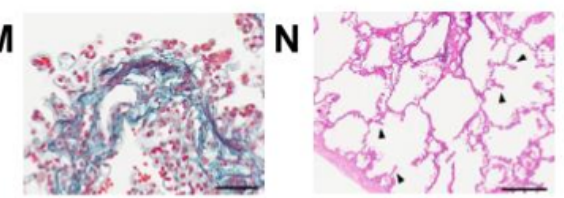